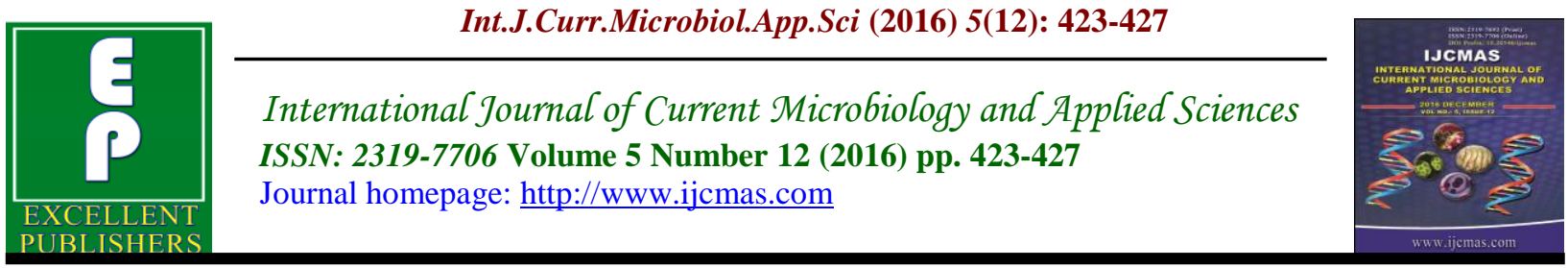

Original Research Article

http://dx.doi.org/10.20546/ijcmas.2016.512.046

\title{
Comparison of Performance of ELISA with Indirect Immunofluoresence for the Testing of Antinuclear Antibodies
}

\author{
J. Euphrasia Latha ${ }^{1}$, Dhason Therese Mary ${ }^{1}$, Kavitha Mohanasundaram ${ }^{1,2} *$ \\ and Sankaralingam Rajeswari ${ }^{1}$ \\ ${ }^{1}$ Institute of Rheumatology, Madras Medical College, India \\ ${ }^{2}$ Institute of Rheumatology, Saveetha Medical College Hospital, Thandalam, India \\ *Corresponding author
}

\section{A B S T R A C T}

Keywords

ELISA, Immunofluoresence and immunoblot.

\section{Article Info}

Accepted:

18 November 2016

Available Online:

10 December 2016
Antinuclear antibody testing is one of the major components of immunological investigations. It is very useful in diagnosing autoimmune connective tissue disorders. ELISA, Immunofluoresence and immunoblot are certain investigations by which ANA testing is done. Indian studies about the performance of ANA testing using ELISA and Immunofluoresence are very less and fail to provide concrete evidence. Universally IF has been accepted as gold standard investigation for ANA testing. In India due to cost constraints physicians still use ELISA testing. In this study we aim to look into the performance of ELISA and IF in the testing of ANA testing.

\section{Introduction}

Antinuclear antibodies (ANA) are antibodies directed against multiple nuclear antigens, often found in patients with autoimmune connective tissue disorders (Slater et al., 1996). Testing of ANA is done by Enzyme Linked Immuno Sorbent Assay (ELISA) and Indirect immunofloresence (IIF) using hep 2 cells (Meroni et al., 2010). ANA testing is used inappropriately in many subjects and may result in wrong diagnosis and subsequent management. Indirect Immunofluoresence has long been accepted as gold standard for ANA testing and current recommendations too address the same (Solomon et al., 2002; Buyon et al., 1993). However, clinicians and laboratories still continue to use the ELISA methodology for ANA testing, in view of easy availability and feasibility. A test to be used for screening should essentially have a good sensitivity and specificity. In this study we compare the two techniques used for ANA testing in our subjects and analyse the results.

\section{Materials and Methods}

The study was done in Institute Of 
Rheumatology, Madras Medical College between January 2015 and June 2015. All patients attending rheumatology outpatient were screened by rheumatologists and patients suspected to have autoimmune connective tissue disorder were requested to undergo a complete evaluation comprising complete blood count, erythrocyte sedimentation rate, blood sugar, renal function and liver function test. Appropriate immunological investigations were asked for - Anti nuclear antibodies, complements C 3, C 4, C Reactive Protein, Rheumatoid Factor, ANA profile, as and when needed. Complements were done using Single Radial Immuno Diffusion (SRID) and CRP, RF was done using semi quantitative latex agglutination method. Autoimmune connective tissue disorders included in our study were systemic lupus erythematosus, sjogren's syndrome, scleroderma, inflammatory myositis, mixed connective tissue disorder and overlap syndrome. Rheumatoid arthritis, hashimoto's thyroiditis and primary liver disorders like autoimmune hepatitis, primary biliary cirrhosis were excluded from the study.

In the immunology lab samples of Anti nuclear antibodies were tested both with ELISA and Hep 2 cell line by Indirect immunofluoresence methodology. For Hep 2 testing 1:80 was the standard dilution used and end dilution titre was done only if indicated (like in MCTD). Pattern reporting was done on the same day using immunofluoresence microscope with LED as light source by independent trained microbiologist. Cytoplasmic pattern positivity was included under ANA positivity. Samples which tested positive for ANA by ELISA or Hep 2 were tested for ANA profile using line immunoassay (Immunoblot) by EUROIMMUNE. Immunoblot was also done in some patients with ANA negativity, where a rheumatologist strongly suspect the presence of immunological disorders like Sjogren's syndrome in the patient and request for the same. Detailed clinical history and final diagnosis of the patient was recorded from the rheumatologist records. ANA testing was also done on age and sex matched healthy controls.

\section{Statistical Analysis}

It was done using SPSS 16 software, sensitivity, specificity, positive predictive and negative predictive value along with likelihood ratios were calculated. The confidence interval of predictive values was calculated using Clopper-Pearson confidence intervals and for the likelihood ratios log method was used.

\section{Results and Discussion}

A total of 150 patients were screened for autoimmune connective tissue disorder and 60 healthy age and sex matched controls underwent anti nuclear antibody testing.

Out of 150 patients after correlating their clinical, hematological and immunological profile 126 patients were found to have autoimmune connective tissue disorder. Out of the 126 patients with AICTD, ANA was positive by Indirect Immunofluoresence in 108 patients and by ELISA it was positive in 86 patients.(Fig 1)

In the remaining 84 subjects (60 healthy controls and 24 subjects tested and declared not to have an AICTD by rheumatologist), ANA positivity by IIF was observed in 6 patients and ELISA positivity in 20 subjects (Table 1).

The 18 patients with negative ANA by IIF were analysed in detail. 3 of them had features suggestive of Sjogren's syndrome, 
12 had features suggestive of idiopathic inflammatory myositis and 3 had features suggestive of scleroderma. The 3 patients with suspected primary Sjogren's Syndrome underwent ANA profile analysis using immunoblot which showed a strong anti SS A positivity. Lip biopsy and Schirmer's tests were also suggestive of pSS in those three patients. Immunoblot done on the other 15 patients was negative. Immunoblot of 6 controls who tested positive for ANA by IIF were negative.

The sensitivity, specificity, positive predictive value and negative predictive value of ANA testing with ELISA and IIF was analysed and results given in Table 2 .

ANA testing is an essential test in diagnosing autoimmune connective tissue disorders like SLE, Sjogrens's syndrome, MCTD, Scleroderma and IIM. ANA by hep 2 testing has been the gold standard investigation for the same (Meroni et al.,
2010). Hep 2 cells are spindle shaped cells derived from human epithelial cell line of laryngeal carcinoma. Since it is of human origin, it replicates all antigens in a human cell and also has a greater yield due to higher mitotic rate (Kavanaugh et al., 2000; Wayne, 1996). More than 20 patterns can be observed in Immunoflurosence and it represents more than 100 antigens. In contrast most ELISA kits are coated with fewer antigens (around 20), both recombinant and human antigens are used, resulting in greater likelihood of false negativity.

ANA negative lupus was previously a distinct entity. With increasing usage of hep 2 cells and better techniques, its now more likely a myth. False negativity in ANA hep 2 testing is rare; however SS A antigens are under represent in hep 2 slides due to difference in methodology of extraction. SSA antigens are susceptible to acetone extraction and hence degraded easily.

Table.1 ANA negativity in ELISA vs IIF

\begin{tabular}{|l|l|l|}
\hline ANA by IF & $\begin{array}{l}\text { True Positive } \\
108\end{array}$ & $\begin{array}{l}\text { False Positive } \\
6\end{array}$ \\
\hline ANA by ELISA & 86 & 20 \\
\hline ANA by IF & False Negative & True Negative \\
& 18 & 78 \\
\hline ANA by ELISA & 40 & 64 \\
\hline
\end{tabular}

Table.2 Sensitivity, specificity, PPV, NPV of ELISA and IIF for ANA testing

\begin{tabular}{|c|c|c|}
\hline & ANA by IF & ANA by ELISA \\
\hline Sensitivity & $85.71(78.37$ to $91.31 \%)$ & $68.25(59.37$ to 76.26$) \%$ \\
\hline Specificity & 92.86(85.10 to $97.33 \%)$ & $76.19(65.65$ to 84.81$) \%$ \\
\hline Positive predictive Value & $94.74(88.90$ to 98.04$) \%$ & $81.13(72.38$ to 88.08$) \%$ \\
\hline Negative Predictive Value & $81.25(72$ to 88.49$) \%$ & $61.54(51.49$ to 70.91$) \%$ \\
\hline Positive Likelihood ratio & $12(5.53$ to 26.03$)$ & $2.87(1.92$ to 4.28$)$ \\
\hline Negative Likelihood ratio & $0.15(0.10$ to 0.24$)$ & $0.42(0.31$ to 0.55$)$ \\
\hline
\end{tabular}


Fig.1 ANA positivity in ELISA vs IF in healthy controls and AICTD's
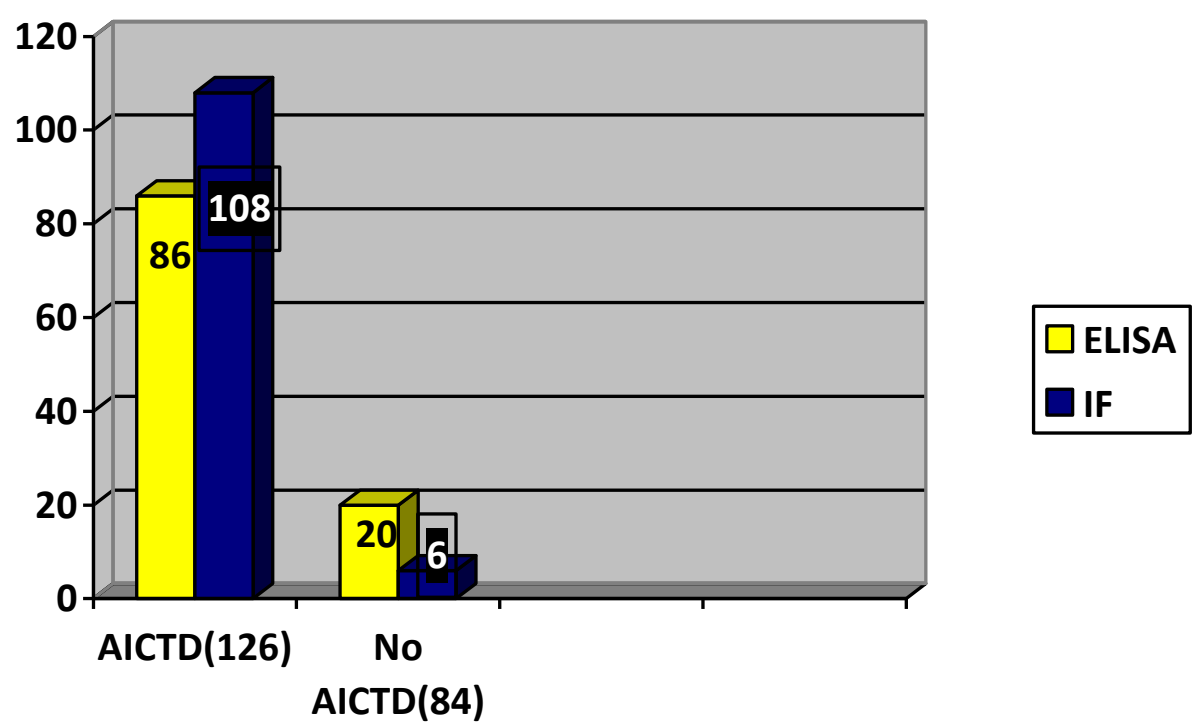

Hence, though rare, patients with Sjogren's syndrome, mothers with lupus with recurrent congenital heart blocks in fetus might show a false ANA negativity in hep 2 testing.

Immunoblot technique might show a positive anti SS A and anti SS B. In our study the 3 patients with Sjogren's syndrome showed false negativity with IIF; myositis specific and myositis associated antigens(MSA's and MAA's respectively) are often under represent in hep 2 cells; hence giving a negative ANA (Love et al., 1991; Imbert-Masseau et al., 2003). Conventional ANA immunoblots does not include MSA and MAA's barring anti Jo 1 and PM Scl (Stone et al., 2007). Immunoblots for MAA and MSA are now available,; though not routinely used in clinical practice since they only have prognostic significance and not diagnostic value. ANA negativity in the setting of a high clinical suspicion of an AICTD warrants an immunoblot analysis, in most cases no further testing is indicated (Thomson et al., 2001).

In conclusion, ANA by indirect immunofluoresence is the gold standard for
ANA testing because of its better sensitivity, specificity, PPV and NPV. We recommend that ELISA should not be used even as a screening procedure even though it is cheaper. In clinical scenarios where likelihood of pSS is high. Even if ANA hep 2 testing is negative ANA immunoblot may be done; otherwise ANA immunoblot is not routinely indicated in negative samples.

\section{References}

Buyon, J.P., Winchester, R.J., Slade, S.S. 1993. Identification of mothers at risk for congenital heart block and other neonatal lupus syndromes in their children. Arthritis Rheum., 36: 12631273.

Cross, L.S., Aslam, A., Misbah, S.A. 2004. Antinuclear antibody-negative lupus as a distinct diagnostic entity - does it no longer exist? Q. J. Med., 97: 303308.

Imbert-Masseau, A., Hamidou, M., Agard, C., et al. 2003. Antisynthetase syndrome, Joint Bone Spine, 70: 161168.

Kavanaugh, A., Tomar, R., Reveille, J., et 
al. 2000. American College of Pathologists: Guidelines for clinical use of the antinuclear antibody test and tests for specific autoantibodies to nuclear antigens, Arch. Pathol. Lab. Med., 124: 71-81.

Love, L.A., Leff, R.L., Fraser, D.D., et al. 1991. A new approach to the classification of idiopathic inflammatory myopathy: myositisspecific autoantibodies define useful homogeneous patient groups, Med., 70: 360-374.

Meroni, P.L., Schur, P.H. 2010. ANA screening: an old test with new recommendations. Ann. Rheum. Dis., 69(8): 1420-1422.

Meroni, P.L., Schur, P.H. 2010. ANA screening: an old test with new recommendations, Ann. Rheum. Dis., 69: 1420-1422.

Reichlin, M. 2000. ANA negative systemic lupus erythematosus sera revisited serologically. Lupus, 9(2): 116-119.

Slater, C.A., Davis, R.B., Shmerling, R.H. 1996. Antinuclear antibody testing. A study of clinical utility. Arch. Intern.
Med., 156(13): 1421-1425.

Solomon, D.H., Kavanaugh, A.J., Schur, P.H. 2002. American College of Rheumatology Ad Hoc Committee on Immunologic Testing Guidelines: Evidence-based guidelines for the use of immunologic tests: Antinuclear antibody testing. Arthritis Rheum., 47: 434-444.

Stone, K.B., Oddis, C.V., Fertig, N., et al. 2007. Anti-Jo-1 antibody levels correlate with disease activity in idiopathic inflammatory myopathy, Arthritis Rheum., 56: 3125-3131.

Thomson, K.F., Murphy, A., Goodfield, M.J., et al. 2001. Is it useful to test for antibodies to extractable nuclear antigens in the presence of a negative antinuclear antibody on Hep-2 cells? J. Clin. Pathol., 54: 413.

Wayne, Pa. 1996. Quality assurance for the indirect immune fluorescence test for auto antibodies to nuclear antigen (IFANA): approved guideline, National Committee for Clinical Laboratory Standards.

\section{How to cite this article:}

Euphrasia Latha, J., Dhason Therese Mary, Kavitha Mohanasundaram and Sankaralingam Rajeswari. 2016. Comparison of Performance of ELISA with Indirect Immunofluoresence for the Testing of Antinuclear Antibodies. Int.J.Curr.Microbiol.App.Sci. 5(12): 423-427. doi: http://dx.doi.org/10.20546/ijcmas.2016.512.046 